\title{
Simulations of disks in binary systems using parallelized SPH to reach extreme spatial resolution
}

\author{
Molteni D., Fauci F., Gerardi G., Valenza M.A. \\ Dipartimento di Fisica e Tecnologie Relative, Universita' di Palermo, \\ Italy
}

\begin{abstract}
Results of 3D numerical simulations of the gas transfer in close binary systems show that it is possible the production of accretion streams having low specific angular momentum in a region close to the accreting star. These streams are mainly placed above the orbital disc. The eventual formation of such bulges and shock heated flows is interesting in the context of advection dominated solutions and for the explanation of spectral properties of the Black Hole candidates in binary systems. We set up a parallelized version of $3 \mathrm{D}$ S.P.H. code, using domain decomposion. with increasing spatial resolution around the compact star.
\end{abstract}

\section{Introduction}

The energy resource of high energy radiation emission from binary systems, containing a collapsed star (white dwarf, neutron star or black hole), is mainly due to the accretion process. The basic mechanism of mass transfer in these binary systems is essentially clear. There are two wide classes of mechanisms: one by wind and one by Roche lobe overflow (ROLOF, cfr. Frank, King \& Raine 1992). The wind process usually is operating in systems containing a high mass normal star, while ROLOF works for normal stars filling their Roche lobe (semidetached binaries). The wind process produces an accretion flow with a small angular momentum. The usual view for the ROLOF model considers the formation of a stream from the inner Lagrangian point $L_{1}$ and the subsequent formation of an accretion disc around the compact star.

\section{Our simulations}

The binary system has stars with equal mass. Details are give in Molteni et al. 2001. Here we present results from SPH (Smoothed Particles Hydrodynamics) simulations. We performed2D and 3D simulations with same parameters. The 2D simulations are used to make a subsequent close comparison of the gas flow with the $3 \mathrm{D}$ case. In $2 \mathrm{D}$ simulations we have typically 25000 particles. 


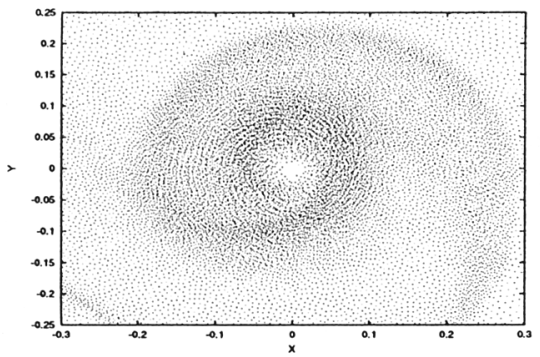

Figure 1. Inner part of $2 \mathrm{D}$ disk simulation.

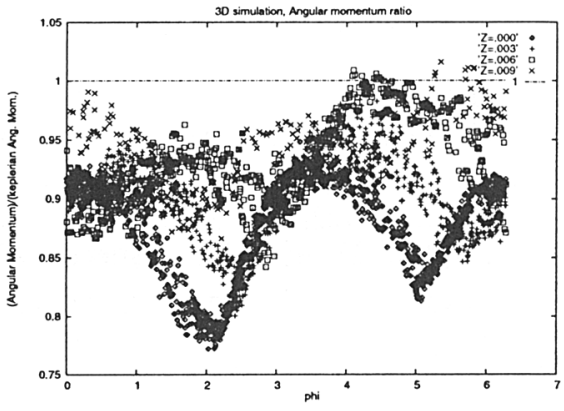

Figure 2. Kepler ratios for particles at different $\mathrm{Z}$ levels.

Fig. 1 shows a zoom of the $2 \mathrm{D}$ disk simulation. It is clearly visible the spiral wave.

In the 3D simulation we have 300.000 particles. Fig.2 shows the kepler ratios for the particles at different $\mathrm{Z}$ levels. It can be seen that the greatest subkeplerian values are obtained for parcels of the fluid above the equator. The $3 \mathrm{D}$ code was efficiently run on a cluster of workstation under MPI software.

\section{Conclusions}

We have shown that, even in compact binary system, is possible the formation of relevant subkeplerian flows accreting onto the stellar compact object. This fact is particularly important for accretion on black hole candidates. Nearly all models of accretion disks around black hole require the formation of a hot bulge comptonizing the canonical disk emission. The origin of this bulge or corona is not clear also for the models that explain well the spectral characteristics in the transient $\mathrm{BH}$ candidates. We want to point the attention to a realistic process that can produce the subkeplerian flows: they don't spring out from the thin standard keplerian disc, but they originate from matter already present and recirculating in the wide potential well of the binary system or in the Roche lobe. Even a much smaller amount of this flow can have significant effects on the spectra of the radiation emitted (Chakrabarti \& Titarchuck, 1995). More detailed physical ingredients have to be considered such as realistic cooling and higher spatial resolution is required. The intensity and the therefore the relevance of this subkeplerian flows will be systematically studied with further $3 \mathrm{D}$ time dependent simulations in progress.

\section{References}

Frank J., King A., Raine D., 1992, Accretion Power in Astrophysics, Cambridge Univ. Press, Cambridge

Chakrabarti S.K., Titarchuk L.G., 1995, ApJ, 455, 623

Molteni, D., Kuznetsov, O. A., Bisikalo, D. V., Boyarchuk, A. A., 2001,MNRAS, 327,1103 